\title{
Intravesical foreign body-induced bladder calculi resulting in obstructive renal failure
}

\author{
Fadi Kamal, MD; ${ }^{*}$ Aaron T.D. Clark, MD; ${ }^{*}$ Luke Thomas Lavallée, BSc;† Matthew Roberts, MD; \\ James Watterson, MD*
}

\begin{abstract}
We report the case of a 30-year-old man who presented with obstructive renal failure and urosepsis due to bladder outlet-obstructing bladder calculi that formed around 3 copper wires that were self-inserted into his urinary bladder 15 years previously. We present the evaluation, imaging and management of the unique complications resulting from the self-insertion of an intra-vesical foreign body. Our patient's case was unique for 2 reasons. First, the length of time (15 yr) from foreign body insertion to presentation is the longest interval reported in the literature. Second, this is the first report of bladder calculi induced by the insertion of a foreign body that resulted in obstructive renal failure.
\end{abstract}

CUAJ 2008;2(5):546-8

\section{Introduction}

A number of case reports in the literature discuss foreign bodies inserted in the lower urinary tract and external genitalia of men. Most cases of self-insertion of foreign bodies are a result of autoeroticism, inquisitiveness or therapeutic or psychiatric illness. ${ }^{1}$ Most patients avoid medical attention out of shame and present later with complications due to the foreign body, including lower urinary tract symptoms, voiding difficulty, hematuria, pain or swelling, extravasation, urinary tract infections or abscess formation.

\section{Case report}

A 30-year-old man presented to the emergency department with a 1 -week history of fatigue. His symptoms progressed over a period of 24 hours to include diaphoresis, nausea, vomiting, fever, shortness of breath and suprapubic pain. At the time of admission, he initially denied having urinary tract symptoms. However, on further questioning he revealed long-standing obstructive symptoms including decreased urinary stream and prolonged voiding time. He had no history of gross hematuria. His medical history included mild hypertension, which was not actively treated. He had no history of urinary tract infections, sexually transmitted infections or urinary tract calculi. Initial investigations revealed leukocytosis $\left(15.2 \times 10^{9} / \mathrm{L}\right)$, elevated creatinine levels $(762 \mu \mathrm{mol} / \mathrm{L})$, hyperkalemia $(\mathrm{K}=6.1)$ and positive urinalysis for red blood cells, protein and nitrites. Blood and urine cultures were negative.
Computed tomography of the abdomen and pelvis revealed severe bilateral hydroureteronephrosis with perinephric fat stranding and advanced renal cortical thinning (Fig. 1). The urinary bladder showed detrusor hypertrophy and contained 3 large bladder calculi obstructing the bladder neck (Fig. 2). Foley catheter insertion required bedside cystoscopic guidance, at which time the patient admitted to having inserted 3 copper wires into his bladder 15 years earlier. A subsequent pelvic radiograph revealed that the bladder calculi appeared to have developed over linear radio-opacities representing the copper wires (Fig. 3)

The patient was admitted to the intensive care unit for management of urosepsis, renal failure and metabolic acidosis. He was started on broad-spectrum antibiotics and required short-term hemodialysis. Subsequent management in the urology ward included

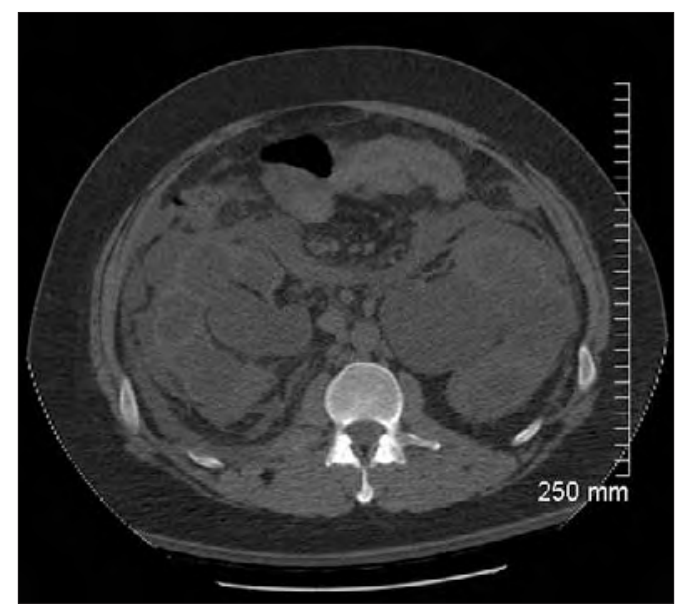

Fig. 1. Computed tomography scan of the abdomen and pelvis revealing severe bilateral hydroureteronephrosis with perinephric fat stranding and advanced renal cortical thinning. 
correction of anemia and controlling sepsis. The patient underwent cystoscopy, which showed 3 large, freely mobile bladder calculi. We removed the calculi by subsequent open cystolithotomy. We closed the bladder in 2 layers, leaving an indwelling urethral catheter and a perivesical drain. The calculi had a combined weight of $95 \mathrm{~g}$.

The patient had an uncomplicated postoperative recovery. One of the removed stones that calcified over the inserted copper wire is shown in Figure 4. After fragmenting the stone, the copper wire, measuring about $6 \mathrm{~cm}$ in length, became visible. Analysis showed that the bladder calculi were composed primarily of calcium carbonate phosphate with a trace of magnesium ammonium phosphate. The patient's creatinine levels decreased to a baseline of $166 \mu \mathrm{mol} / \mathrm{L}$ with relief of the obstruction. A follow-up voiding cystogram showed no leakage with good bladder emptying. We did not request a psychiatric assess-

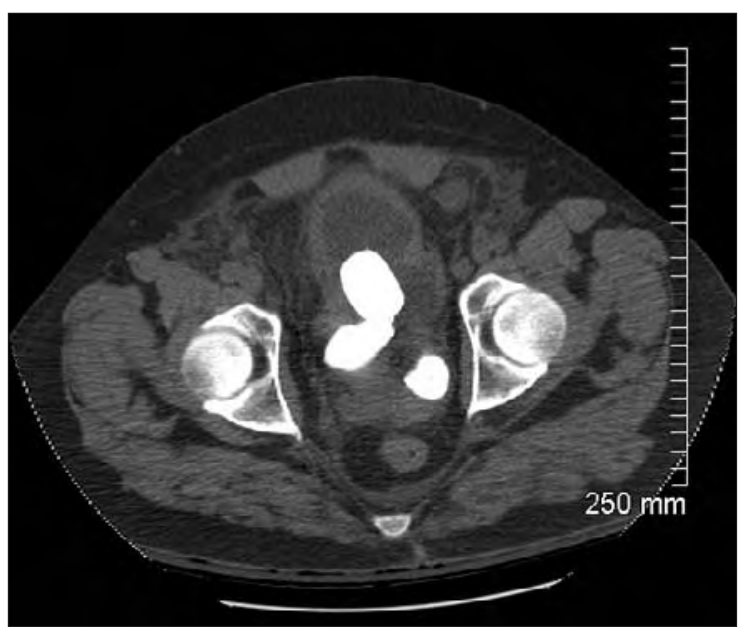

Fig. 2. Detrusor hypertrophy and 3 large bladder calculi obstructing the bladder neck.

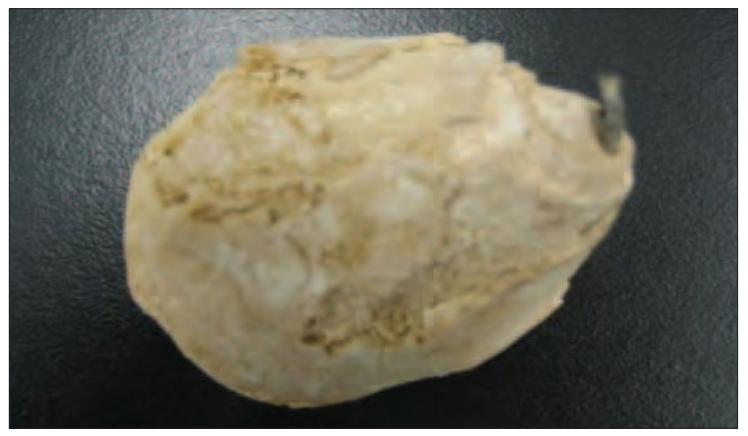

Fig. 4. Bladder stone calcified over the copper wire. ment for our patient because he had no apparent psychiatric illness. His teenage experience was most likely a form of sexual stimulation.

\section{Discussion}

Risk factors for bladder calculi include urinary stasis, infection and the presence of foreign bodies. Typical presentations include bladder outlet obstruction, urinary tract infection, terminal hematuria, intermittent pain and irritating urinary symptoms such as frequency, urgency and dysuria.

Foreign bodies may be introduced into the bladder iatrogenically, via migration from adjacent organs, by penetrating injuries or, as in our patient, by self-insertion, which is usually a result of eroticism, inquisitiveness or psychiatric illness. ${ }^{1}$ In cases of self-insertion, psychiatric evaluation has been suggested. ${ }^{2,3} \mathrm{~A}$ multitude of foreign bodies, including

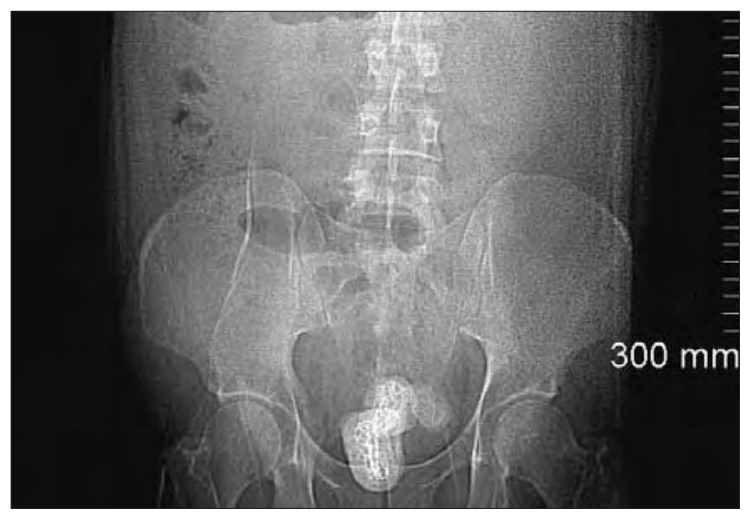

Fig. 3. Pelvic radiograph revealing bladder calculi that developed over linear radio-opacities.

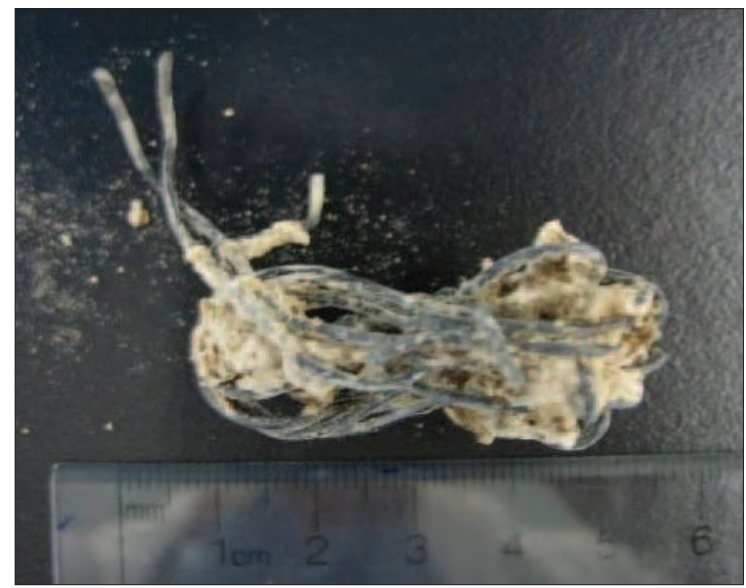

Fig. 5. Copper wire, the nidus for bladder stone formation. 
Kamal et al.

intrauterine devices, medical sutures, hair, wires and artificial urinary sphincters, have been associated with bladder calculi. ${ }^{1,2,-9}$

In our patient, bladder calculi induced by the presence of a foreign body were the result of selfinsertion. Our patient's case was unique for several reasons. First, the mild obstructive urinary symptoms and the absence of severe infection for 15 years were surprising given the number of intravesical foreign bodies and the size of the calculi at presentation. Second, it demonstrates how patients with foreign bodies can progress to lifethreatening acute renal failure, thus indicating the importance of definitive management with all vesical foreign bodies. Urological history taking had to be stressed to confirm the presenting symptoms because our patient was embarrassed to reveal his urinary history. To our knowledge, no other cases have been reported of foreign body-induced bladder calculi leading to acute renal failure.

\section{Conclusion}

Foreign body-induced bladder calculi are a diagnostic and therapeutic challenge. Our patient's case demonstrated an atypical and serious presentation that can result years after initial foreign body placement.
From the *Division of Urology, Department of Surgery, University of Ottawa, Ottawa, Ont. and the TFaculty of Medicine, McGill University, Montréal, Que.

This article has been peer reviewed.

Competing interests: None declared.

\section{References}

1. Loeser A, Gerharz EW, Riedmiller H. Chronic perforation of the urinary bladder by self-inserted foreign body. Int Urogynecol J Pelvic Floor Dysfunct 2007; 18:689-90.

2. Kenney RD. Adolescent males who insert genitourinary foreign bodies: Is psychiatric referral required? Urology 1988;32:127-9.

3. Costa G, Di Tonno F, Capodieci S, et al. Self-introduction of foreign bodies into the urethra: a multidisciplinary problem. Int Urol Nephrol 1993;25:77-81.

4. Bartoletti $R$, Gacci $M$, Travaglini $F$, et al. Intravesical migration of AMS 800 artificial urinary sphincter and stone formation in a patient who underwent radical prostatectomy. Urol Int 2000;64:167-8.

5. Huang WC, Yang JM. Sonographic appearance of a bladder calculus secondary to a suture from a bladder neck suspension. I Ultrasound Med 2002;21:1303-5.

6. Derry P, Nuseibeh I. Vesical calculi formed over a hair nidus. Br J Urol 1997;80:965.

7. Khan ZA, Khan SA, Williams A, et al. Intravesical migration of levonorgestrel-releasing intrauterine system (LNG-IUS) with calculus formation. Eur J Contracept Reprod Health Care 2006;11:243-5.

8. Nazir SS, Wazir BS, Qazi S. An unusual foreign body in the urinary bladder. JK Pract 2004;11:268-9

9. Bird VG, Winfield HN. Removal of bladder stone with metal wire infrastructure. Can J Urol 2002;9:1500-2

Correspondence to: Dr. Fadi Kamal, The Ottawa Hospital, Civic Campus, Department of Urology, B3, 1053 Calring Ave., Ottawa ON KIY 4E9; fadkam@gmail.com 